\title{
Avaliação dos processamentos de crosstalk e de correção atmosférica em dados ASTER para identificação da relação mineralógica caulinita/(caulinita + gibbsita) em solos no município de São João d’Aliança (GO)
}

\author{
Evaluation of atmospheric and crosstalk's correction in ASTER data \\ for the identification of kaolinite/(kaolinite+gibbsite) mineralogical \\ ratio in soils of São João d'Aliança (GO)
}

\section{Daniella Dias Vivaldi $^{1 *}$, Gustavo Macedo de Mello Baptista ${ }^{1}$, Paulo Roberto Meneses ${ }^{1}$}

\begin{abstract}
RESUMO: Este estudo teve por objetivo avaliar a influência das correçóes crosstalk e atmosférica em imagens ASTER-SWIR na identificação da relaçáo caulinita/(caulinita + gibbsita) em solos tropicais. Essa relação é representada pelo índice espectral $\mathrm{RCGb}_{\text {cale }}$. Foram utilizados os dados L1A e L1B e os produtos sob demanda AST_07 e AST_07XT, de 24 de outubro de 2001, os módulos de correção atmosférica FLAASH e QUAC e o programa de correçấo crosstalk CRSTK30. Os resultados foram analisados segundo quatro abordagens: comparaçấo do comportamento dos transectos do RCGb sele $_{\text {e }}$ do $\mathrm{RCGb}_{\mathrm{ATG}}$, análise da imagem RCGb $\mathrm{s}_{\text {scale }}$ fatiada em classes, análise da imagem $\mathrm{RCGb}_{\text {sale }}$ e análise da similaridade estatística entre os valores dos transectos do $\mathrm{RCGb}_{\text {sale }}$ e do $\mathrm{RCGb}_{\mathrm{ATG}}$, por meio do teste estatístico de Mann Whitney. A aplicação dos módulos FLAASH e QUAC prejudicou a identificação dos ambientes e da relação mineralógica. A combinação CRSTK30-FLAASH prejudicou a identificação dos ambientes, da relação mineralógica e do comportamento do transecto do RCGb $b_{\text {scale }}$. A combinaçáo CRSTK30-QUAC forneceu melhor resultado de similaridade estatística em relaçáo aos resultados dos processamentos sem correçóes. De acordo com a classificaçáo das análises, conclui-se que, para a investigação da relaçấo caulinita/(caulinita+gibbsita) na área de estudo, o produto sob demanda AST_07 e a imagem de reflectância L1B, com correçáo crosstalk e com correçáo atmosférica, por meio do módulo QUAC, são as mais adequadas, nessa ordem.
\end{abstract}

PALAVRAS-CHAVE: ASTER SWIR; correção atmosférica; crosstalk; índice espectral RCGb $b_{\text {scale }}$; São João d'Aliança.

\begin{abstract}
The aim of this study was to analyze the influence of atmospheric correction and crosstalk in the spatial relationship of kaolinitel(kaolinite + gibbsite) in tropical soils through the application of the spectral index RCGb, in ASTER-SWIR L1A and L1B images, and in products on-demand AST_07 and AST_07XT, acquired on October 24, 2001. Atmospheric correction routines FLAASH and QUAC and the CRSTK30 software were used to correct for the crosstalk effect. The results were analyzed according to four approaches: comparing the behavior of RCGb and RCGb transects, analysis of the $R C G b_{\text {sale }}$ image of sliced values and analysis of the RCGb image and statistical analysis of the similarity between the values of the $R C G b_{\text {scale }}$ and $R C G b_{A T G}$ transects through the Mann-Whitney statistical test. The routines FLAASH and QUAC hampered the identification of environments and mineralogical relationship. The combination CRSTK30-FLAASH impaired the identification of environments, as well as the relationship mineralogical and the behavior of the $R C G b_{\text {sale }}$ transect. The combination CRSTK30-QUAC provided better results in terms of the statistical similarity in relation to the results of the processing without corrections. The results indicated that the products on-demand AST_07 and images $L 1 B$ converted to reflectance with crosstalk correction and atmospheric correction (QUAC) are the most appropriate, in this order.
\end{abstract}

KEYWORDS: ASTER-SWIR; atmospheric correction; crosstalk, spectral index RCGb sale; São João d'Aliança.

${ }^{1}$ Programa de Pós-graduação Stricto Sensu em Geociências Aplicadas, Universidade de Brasília - UnB, Brasília (DF), Brasil. E-mails: dvivaldi@gmail.com gmbaptista@unb.br,pmeneses@unb.br

*Autor correspondente

Manuscrito ID 29935. Recebido em: 15/03/2013. Aprovado em: 26/08/2013. 


\section{INTRODUÇÃO}

Uma das aplicaçóes do sensoriamento remoto refere-se ao mapeamento de relaçóes mineralógicas nos solos tropicais a fim de identificar o grau de intemperismo (Baptista 2006).

Embora a resposta espectral do solo seja influenciada por vários fatores que atuam em conjunto (matéria orgânica, óxidos de ferro, fração de argila, umidade, entre outros), a reflectância de certos minerais, em alguns intervalos espectrais, apresenta-se preservada, o que possibilita a identificaçáo remota por meio de dados de alta resolução espectral (Galvão et al. 1997).

Os solos tropicais geralmente apresentam alto grau de intemperismo e, considerando que: (i) os latossolos são formados a partir da remoção de sílica e de bases trocáveis e por posterior acúmulo de alumínio; (ii) o que resta de sílica encontra-se na caulinita e (iii) a gibbsita apresenta, além da sílica, o alumínio, a relação entre os dois minerais possibilita entender o grau de intemperismo das coberturas pedológicas (Baptista \& Madeira Netto 2001).

Em geral, as técnicas de classificação usadas para identificaçáo mineral por meio de sensoriamento remoto envolvem a comparação da similaridade dos espectros de reflectância, extraídos diretamente das imagens, com espectros de referência dos minerais (amostras de campo ou bibliotecas espectrais) para sua identificaçáo na cena analisada (Meneses apud Meneses \& Madeira Netto 2003).

A partir do desenvolvimento e da aplicação de alguns índices espectrais, o estudo das relaçóes mineralógicas foi otimizado em tempo e em recursos direcionados ao processo de reconhecimento e mapeamento de solos, o qual dependia, anteriormente, de uma metodologia onerosa (Meneses apud Meneses \& Madeira Netto 2003).

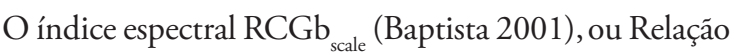
Caulinita/Gibbsita a partir das imagens scale, produtos resultantes da aplicação da classificação Spectral Feature Fitting, é entendido como índice proporcional à relação molecular sílica-alumina ou valor Ki dos solos.

Baptista (2001) desenvolveu o índice RCGb scale $_{\text {a partir }}$ de dados do sensor hiperespectral Airborne Visible InfraRed Imaging Spectrometer (AVIRIS) do Jet Propulsion Lab/NASA (JPL/NASA), os quais forneceram resultados muito próximos aos dados obtidos em campo pelo autor, isto é, os resultados se mostraram eficazes no mapeamento da relação mineralógica, proporcionando uma quantificação significativa do grau de intemperismo dos solos estudados nos municípios goianos de São João d'Aliança e Niquelândia.

No entanto, ao testar o índice RCGb scale $_{\text {em dados Advanced }}$ Spaceborne Thermal Emission and Reflection Radiometer (ASTER)Short Wave Infrared (SWIR), Baptista et al. (2007) perceberam o deslocamento das feiçôes de absorção dos minerais caulinita e gibbsita, bem como o ponto de máxima reflexão entre suas feiçôes. Esse deslocamento está relacionado ao efeito crosstalk, entendido como um "vazamento" de radiação dos detectores da banda 4 para os detectores das bandas vizinhas 5 e 9; porém, até então, não se sabia ao certo o quanto esse deslocamento prejudicava a identificação da relação mineralógica.

Nesse contexto, esse estudo teve por objetivo avaliar os processamentos de crosstalk e de correção atmosférica em dados ASTER-SWIR para identificação da relação mineralógica caulinita/(caulinita + gibbsita) em solos no município de São João d'Aliança (GO), e assim dirimir dúvidas a respeito da eficiência das imagens anteriormente observadas.

\section{Área de estudo}

A área de estudo encontra-se no município de São João d'Aliança, localizado na porção norte do estado de Goiás, próximo à Chapada dos Veadeiros, a aproximadamente 150 km de Brasília (DF). A Fig. 1 apresenta o mapa de localização da área de estudo.

Segundo Faria (1995), no município de Sáo João d'Aliança ocorrem rochas granito-gnáissicas e de unidades sedimentares proterozoicas atribuídas ao Grupo Paranoá. A área de estudo encontra-se sobre a litofácies Metassiltito Argiloso (unidade S) (Baptista 2006).

A porção da imagem investigada caracteriza-se, geomorfologicamente, como o topo de uma vertente convexa com caimento NW-SE, e a espacialização das relaçóes minerais dos solos tende a acompanhar a topografia, ou seja, nas porçôes mais conservadas e menos afetadas pela erosão os solos devem apresentar menor alteração, enquanto as áreas mais intemperizadas devem apresentar uma cobertura de solos mais alterada (Baptista et al. 2007). De acordo com a classificação de solos da Embrapa (1999), a área apresenta Latossolo vermelho-escuro argiloso.

\section{MATERIAL E MÉTODOS}

Os dados L1A e L1B do sensor ASTER-SWIR, os produtos sob demanda AST_07 e AST_07XT, os dados mineralógicos ATG da caulinita e da gibbsita obtidos por Baptista (2001), o programa Crosstalk Correction (CRSTK 30) e os módulos Fast Line-of-sight Atmospheric Analysis of Spectral Hypercubes (FLAASH) e Quick Atmospheric Correction (QUAC) do programa Environment for Visualizing Images (ENVI) constituem o material utilizado neste estudo e formam a base para o processamento das imagens $\mathrm{RCGb}_{\text {scale }}$ para as análises propostas.

\section{Sensor Advanced Spaceborne Thermal Emission and Reflection Radiometer}

O sensor multiespectral ASTER está a bordo da plataforma Terra, produto de cooperação entre a NASA e o 


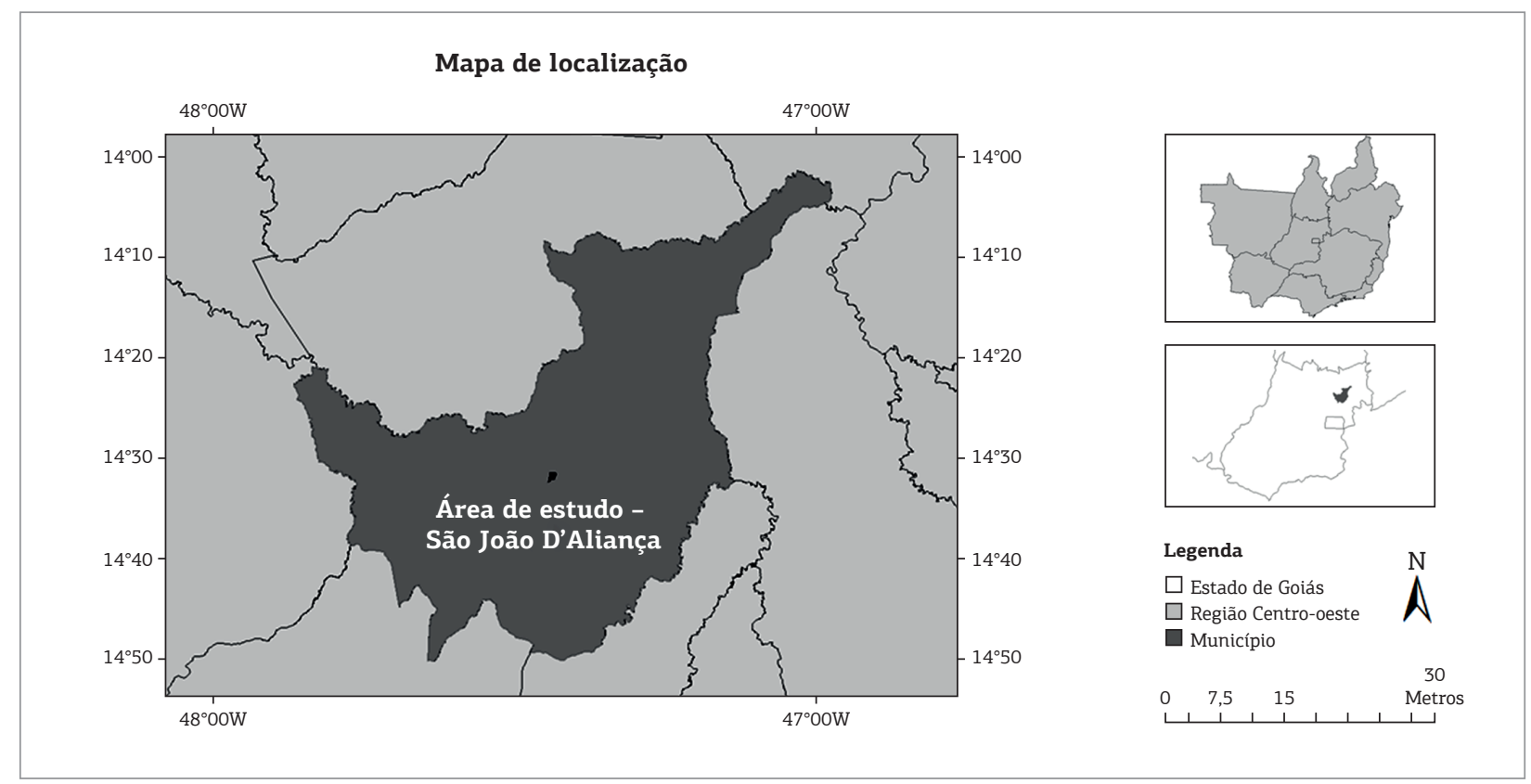

Figura 1. Mapa de localização da área de estudo.

Ministério do Comércio Internacional e Indústria do Japão, lançado em 1999 (Abrams \& Kahle 2000).

O satélite Terra tem órbita circular, quase polar, hélio-síncrona, com altitude de $705 \mathrm{~km}$, com passagem 30 min após o satélite Landsat 7. Cruza o equador às 10 h30 da manhá, hora solar local, em órbita descendente, voltando à mesma órbita a cada 16 dias (Abrams et al. 2007).

O ASTER fornece observaçóes desde a região do visível até a regiâo do infravermelho termal, por meio de 14 bandas espectrais, produzindo cerca de 650 cenas por dia. A justificativa para esse período reduzido de imageamento está na limitação de memória para armazenamento e capacidade de transmissão de dados. Todas as 650 cenas são adquiridas em nível de dados brutos L1A, sendo que, desse total, cerca de 310 sáo dados brutos no nível do sensor L1B, a partir de critério de cobertura de nuvens. Esses produtos são armazenados em arquivos no formato HDF (Hierarchical Data Format) (Abrams et al. 2007).

O ASTER é composto por três subsistemas: Visible and Near Infrared Radiometer (VNIR), SWIR e Thermal Infrared Radiometer (TIR) (Jensen 2009).

O subsistema SWIR opera em 6 bandas espectrais na região de 1,6 $\mu$ m (banda 4) a 2,43 $\mu$ m (banda 9), por meio de um único telescópio apontado ao nadir, com resolução espacial de $30 \times 30 \mathrm{~m}$. Um espelho de apontamento lateral com $\pm 8,5^{\circ}$ recobre as faixas adjacentes à faixa do nadir (Jensen 2009).

O SWIR contém 2.048 PT-SI (platina siliceto) matrizes escalonadas lineares do tipo CCD para cada uma de suas 6 bandas espectrais, espaçadas $1,33 \mu \mathrm{m}$ na ordem em que as bandas se encontram, ou seja, 7, 8, 9, 4, 5 e 6. Na frente de cada par de matrizes CCD existem filtros de interferência que, espectralmente, separam a radiaçáo reflectida provinda da superfície da Terra (USGS 2013). A Tab. 1 apresenta a descrição dos três subsistemas do sensor ASTER.

\section{Dados ASTER - SWIR}

Os dados de radiância L1A não apresentam as calibraçôes dos coeficientes de correçáo geométrica, de calibração radiométrica e dos coeficientes para correçáo do erro de paralaxe. A calibraçáo dos coeficientes de correçáo geométrica consiste de uma série de transformaçóes de coordenadas realizadas em cada uma das bandas. A calibração radiométrica consiste em correçóes a partir de informaçóes de offset e sensibilidade, geradas a partir de uma base de dados para todos os detectores e atualizados periodicamente. A calibração dos coeficientes para correção do erro de paralaxe do SWIR geralmente é feita utilizando técnicas de combinação (matching) ou de dados de modelos numéricos de terreno. O erro de paralaxe do SWIR ocorre devido a um deslocamento no alinhamento do detector (offset), na direção do deslocamento da plataforma, em função da distância entre a plataforma e a superfície observada na Terra (ERSDAC 2003, Abrams et al. 2007).

Os dados L1B apresentam os coeficientes de correção geométrica e radiométrica calibrados no nível do sensor, sendo armazenados em um arquivo de metadados, distribuído no arquivo HDF. As bandas do SWIR e do TIR são registradas em relação ao VNIR e o erro de paralaxe do SWIR encontra-se corrigido. Os dados L1B padrão são gerados na projeção UTM e reamostrados por convolução cúbica. Tanto as imagens L1A, quanto as imagens L1B, são fornecidas em valores de DN (Digital Number) (Abrams et al. 2007). 
Os produtos sob demanda ASTER AST_07 e AST_07XT são fornecidos com a correção atmosférica para as regióes do visível infravermelho próximo (VNIR) e infravermelho de ondas curtas (SWIR). Porém, o produto AST_07 mantém o efeito crosstalk. Cada cena inclui dois arquivos HDF: um para o VNIR e outro para o SWIR (USGS 2013).

O algoritmo do módulo da correção do crosstalk não apresenta simetria à direção transversal da trajetória e, para o ajuste, outras funçóes são incluídas. Os coeficientes de sensibilidade radiométrica são estatisticamente derivados para garantir que uma consistência de calibração seja mantida nas fases pré e pós-correção crosstalk (USGS 2013). A Tab. 2 apresenta os produtos ASTER utilizados neste estudo.

\section{Dados mineralógicos obtidos por meio de Análise Termo-Gravimétrica}

As amostras de solos foram coletadas por meio de tradagem, com espaçamento de $100 \mathrm{~m}$ entre elas com 0 a $20 \mathrm{~cm}$ de profundidade. Foram selecionados 13 pontos seguindo um traçado de $1.300 \mathrm{~m}$ com sentido L-O (Baptista 2006).

A quantificação dos teores de caulinita e de gibbsita foi obtida por meio do método de análise termo-gravimétrica (ATG) (Mackenzie 1982).

Segundo a descrição de Baptista (2006), as amostras passaram pelo procedimento de secagem ao ar e foram peneiradas a $2 \mathrm{~mm}$. A Tab. 3 apresenta os valores ATG dos pontos coletados por Baptista (2001), os quais foram utilizados para a obtenção do RCGb.

Tabela 1. Subsistemas do sensor Advanced Spaceborne Thermal Emission and Reflection Radiometer

\begin{tabular}{|c|c|c|c|c|c|}
\hline Subsistema & Banda & $\begin{array}{c}\text { Faixa } \\
\text { espectral }(\mu \mathrm{m})\end{array}$ & $\begin{array}{l}\text { Comprimento de } \\
\text { onda médio }(\mu \mathrm{m})\end{array}$ & $\begin{array}{c}\text { Resolução } \\
\text { espacial (m) }\end{array}$ & $\begin{array}{l}\text { Resolução } \\
\text { radiométrica (bits) }\end{array}$ \\
\hline \multirow{3}{*}{ VNIR } & 1 & $0,52-0,60$ & 0,556 & \multirow{3}{*}{15} & \multirow{3}{*}{8} \\
\hline & 2 & $0,63-0,69$ & 0,661 & & \\
\hline & 3 & $0,78-0,86$ & 0,807 & & \\
\hline \multirow{6}{*}{ SWIR } & 4 & $1,60-1,70$ & 1,656 & \multirow{6}{*}{30} & \multirow{6}{*}{8} \\
\hline & 5 & $2,145-2,185$ & 2,167 & & \\
\hline & 6 & $2,185-2,225$ & 2,209 & & \\
\hline & 7 & $2,235-2,285$ & 2,262 & & \\
\hline & 8 & $2,295-2,365$ & 2,336 & & \\
\hline & 9 & $2,360-2,430$ & 2,400 & & \\
\hline \multirow{5}{*}{ TIR } & 10 & $8,125-8,475$ & 8,291 & \multirow{5}{*}{90} & \multirow{5}{*}{12} \\
\hline & 11 & $8,475-8,825$ & 8,634 & & \\
\hline & 12 & $8,925-9,275$ & 9,075 & & \\
\hline & 13 & $10,25-10,95$ & 10,657 & & \\
\hline & 14 & $10,95-11,65$ & 11,318 & & \\
\hline
\end{tabular}

VNIR: Visible and Near Infrared Radiometer; SWIR: Short Wave Infrared; TIR:Thermal Infrared Radiometer

Tabela 2. Produtos Advanced Spaceborne Thermal Emission and Reflection Radiometer utilizados neste estudo

\begin{tabular}{c|c|c}
\hline Nome & Produto & Resolução espacial \\
\hline AST L1A & Radiância (dados brutos) & $30 \mathrm{~m}$ \\
\hline AST L1B & Radiância registrada no sensor & $30 \mathrm{~m}$ \\
\hline AST_07 & Reflectância & $30 \mathrm{~m}$ \\
\hline AST_07XT & Reflectância & $30 \mathrm{~m}$ \\
\hline
\end{tabular}


Tabela 3. Valores da Análise Termo-Gravimétrica para São João d'Aliança (Baptista 2001)

\begin{tabular}{|c|c|}
\hline Ponto & ATG \\
\hline SJA p1 $(0-20 \mathrm{~cm})$ & 0,74 \\
\hline SJA p2 $(0-20 \mathrm{~cm})$ & 0,71 \\
\hline SJA p3 $(0-20 \mathrm{~cm})$ & 0,79 \\
\hline SJA p4 $(0-20 \mathrm{~cm})$ & 0,83 \\
\hline SJA p5 $(0-20 \mathrm{~cm})$ & 0,82 \\
\hline SJA p6 $(0-20 \mathrm{~cm})$ & 0,81 \\
\hline SJA p7 $(0-20 \mathrm{~cm})$ & 0,80 \\
\hline SJA p8 $(0-20 \mathrm{~cm})$ & 0,77 \\
\hline SJA p9 $(0-20 \mathrm{~cm})$ & 0,75 \\
\hline SJA p10 $(0-20 \mathrm{~cm})$ & 0,74 \\
\hline SJA p11 $(0-20 \mathrm{~cm})$ & 0,70 \\
\hline SJA p12 (0 - $20 \mathrm{~cm})$ & 0,70 \\
\hline SJA p13 $(0-20 \mathrm{~cm})$ & 0,68 \\
\hline
\end{tabular}

\section{Programa Environment for Visualizing Images}

O programa ENVI disponibiliza ferramentas avançadas, as quais permitem importar, explorar, preparar, analisar e compartilhar informaçóes das imagens geoespaciais de forma ágil e eficiente. É baseado na plataforma IDL (Interactive Data Language), que facilita a expansão e customização da aplicação do programa. O ENVI processa imagens obtidas a partir de inúmeros tipos de satélites e de sensores aerotransportados, incluindo os sensores multiespectrais, como o ASTER (Envi 2009).

\section{Biblioteca Espectral Jet Propulsion Laboratory}

As bibliotecas espectrais são utilizadas como padróes de referência para estudos de solos realizados por meio de dados de sensores multi e hiperespectrais, que visam detectar minerais opticamente ativos. Por meio delas, é possível simular diversas situaçóes, tanto em questóes de monitoramento e análise de alvos terrestres como em questóes referentes aos sistemas de imageamento (Galvão et al. 2007, Baptista 2006).

O ENVI possui três bibliotecas espectrais produzidas pelo JPL, que contêm as características de 160 minerais diferentes no intervalo espectral de 0,4 a 2,5 mm (Envi 2009).

Neste estudo, foram utilizados os espectros de referência Kaolinite Well-Ordered PS-1A e Gibbsite Synthetic OH-3A (Grove et al. 1992). A Fig. 2 apresenta as assinaturas espectrais dos dois minerais investigados.

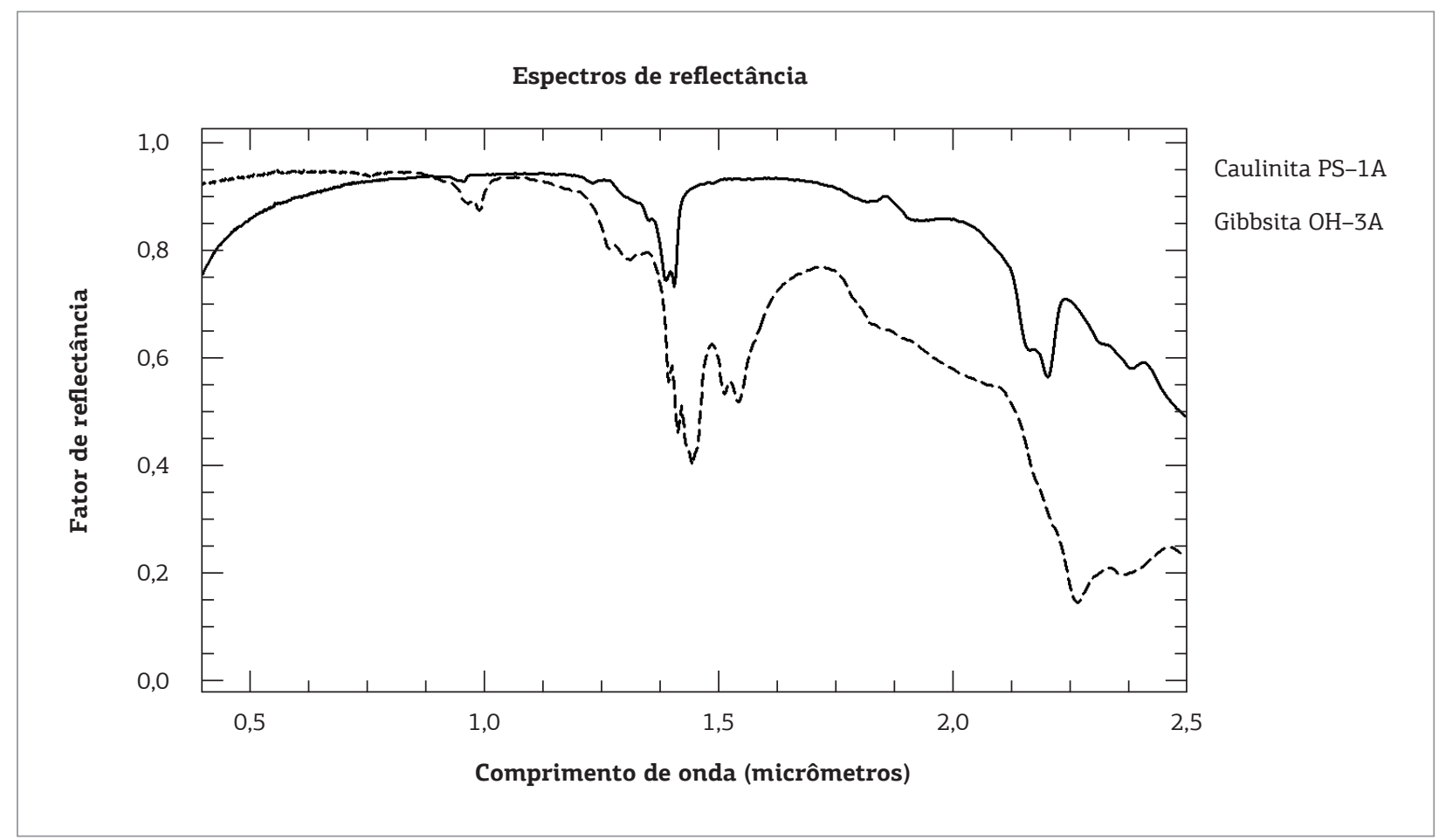

Figura 2. Assinaturas espectrais Kaolinite Well-Ordered PS-1A e Gibbsite Synthetic OH-3A. 


\section{Spectral Feature Fitting}

Algoritmo baseado no nível de absorção que ajusta o espectro dos pixels da imagem ao espectro de referência de uma biblioteca espectral por meio do ajuste por mínimos quadrados (Envi 2009).

O Spectral Feature Fitting (SFF) fornece duas imagens: imagem scale, entendida como a medida da intensidade da feição, e a imagem RMS error, relacionada ao erro médio quadrático. As imagens de erro separadas podem ser utilizadas em dispersogramas bidimensionais para determinar onde determinados materiais ocorrem. As imagens da razão entre os erros mostram a distribuição de cada espectro de referência, em que os melhores ajustamentos aparecem como pixels claros (Envi 2009).

As escalas dos espectros da imagem são redimensionadas para se ajustar aos espectros de referência após a remoção do contínuo de ambos os conjuntos. Em seguida, os espectros da imagem e de referência são comparados dois a dois em determinados valores de comprimento de onda, e o erro médio quadrático é calculado em relação a cada espectro de referência (Envi 2009).

O módulo SFF do ENVI já executa a remoção do contínuo espectral, o que significa que o espectro de radiância ou reflectância do alvo é normalizado para que a comparação de uma feição de absorção, a partir de uma linha de base comum, seja possível. Em termos técnicos, esse procedimento está baseado no ajuste de todo o espectro e utiliza estreitos segmentos de reta conectados aos pontos de máxima reflectância (Baptista 2006).

\section{Flat Field}

Segundo Baptista (2012), a correção atmosférica é muito comum em estudos que abordam o comportamento das feiçôes espectrais. A justificativa está na ideia de reduzir os dados brutos de radiância para dados de reflectância de superfície, para facilitar a identificação das feiçôes espectrais.

Porém, existem alguns procedimentos de pré-processamento que permitem essa transformação sem alterar os efeitos de espalhamento e absorção: é o caso do módulo Flat Field (Baptista 2012).

Esse módulo é definido como uma calibração normalizada, eficaz na redução dos dados do espectrômetro a uma reflectância relativa. $\mathrm{O}$ espectro médio da região de interesse é usado como o espectro de referência, que é dividido pelo espectro de cada pixel da imagem (Baptista 2012, Envi 2009).

\section{Fast Line-of-sight Atmospheric Analysis of Spectral Hypercubes}

FLAASH é um algoritmo desenvolvido pela Spectral Sciences, Inc., sob o patrocínio do Laboratório de Pesquisa da Força Aérea dos EUA, com a finalidade de corrigir os efeitos atmosféricos em imagens de reflectância de sensores hiperespectrais e multiespectrais em comprimentos de onda do visível, do infravermelho próximo, de ondas curtas e das regiôes do infravermelho (Envi 2009).

O programa corrige os efeitos atmosféricos de imagens de sensores multiespectrais adquiridas em qualquer vertical (nadir) ou inclinação geométrica. Para isso, utiliza o código de transferência radiativa MODTRAN4 e executa a correção para o efeito de adjacência (mistura de pixel devido ao espalhamento de superfície), e também calcula a visibilidade média da cena (aerossol/névoa) (Kruse 2004).

O FLAASH opera na faixa espectral entre 0,4 e 2,5 $\mu \mathrm{m}$, e os processamentos são feitos pixel a pixel. O modelo parte da imagem de radiância que chega ao sensor e permite obter a reflectância de superfície a partir da derivação de parâmetros atmosféricos como albedo, altitude da superfície, coluna de vapor e água, profundidade óptica de aerossóis e nuvens, além da temperatura da superfície e atmosfera (Kruse 2004).

\section{Quick Atmospheric Correction}

Módulo de correçáo atmosférica para imagens multiespectrais e hiperespectrais na faixa entre 0,4 a 2,5 $\mu \mathrm{m}$. Esse módulo efetua a correção atmosférica a partir de modelos empíricos utilizando somente as informaçôes contidas na cena, não necessitando de informaçóes auxiliares. $\mathrm{O}$ processamento, significativamente mais rápido, consiste na busca da resposta espectral média dos objetos que compóem a cena (Envi 2009).

\section{Programa Crosstalk Correction}

O SWIR é afetado por um problema de "vazamento" de sinal, o crosstalk, observado após o lançamento do ASTER em 1999.

O problema está, de fato, nos detectores da banda 4, em que a luz incidente é refletida pelos detectores, os quais produzem o "vazamento" sobre os detectores das bandas vizinhas 5 e 9. Além disso, ele é agravado pelo efeito de paralaxe de banda para banda e pela distância entre os pares de matriz CCD. A banda 4, além de ser a de maior amplitude do SWIR, também é a banda com maior potência de refletividade, sendo que a radiação registrada por ela é cerca de quatro a cinco vezes mais intensa que as demais bandas (ERSDAC 2003).

Para tentar minimizar o "vazamento" de sinal, o algorítmo CRSTK30 foi desenvolvido pela ERSDAC, para ser aplicado sobre os dados de entrada do produto de nível L1B do ASTER (ERSDAC 2003).

\section{Índices $\mathrm{RCGb}$ e $\mathrm{RCGb}$ scale}

Em solos que apresentam níveis elevados de intemperismo, observa-se a remoção de sílica assim como o acúmulo de alumínio. A caulinita apresenta em sua composição química a sílica e a alumina, e a gibbsita apresenta alumina (Baptista 2006). 
Madeira Netto desenvolveu o índice da relação caulinita/ gibbsita (Ki), considerando os pontos de reflectância mínima da caulinita e da gibbsita centrados, respectivamente em 2,205 e 2,265 $\mu \mathrm{m}$ em relação ao ponto de máxima reflectância em 2,225 m (Madeira Netto apud Baptista 2006).

Baptista et al. apresentaram um estudo com resultados que mostravam a viabilidade do mapeamento da relação sílica/alumina (IKi), por meio dos dados AVIRIS, em solos tropicais. Porém, os índices espectrais RCGb e RCGb $b_{\text {scale }}$ foram desenvolvidos com a finalidade de solucionar a presença de valores negativos observada na escala dos valores de $\mathrm{Ki}$, o que poderia levar a uma interpretação equivocada (Baptista et al. apud Baptista 2001).

A razão entre as imagens scales e RMS fornece as fit images, que indicam a possibilidade dos materiais dos espectros de referência, os endmembers, existirem na cena. No entanto, as fit images não podem ser comparadas com o resultado do $\mathrm{IKi}$, pois este fornece a quantificação da relação molecular sílica-alumina, enquanto que as fit images indicam a probabilidade de se ter o endmember na cena (Baptista 2006).

Assim, o RCGb tem os mesmos fundamentos do índice IKi. A única diferença está na determinaçâo de Ikaol e Igib baseada no procedimento de razão de três bandas de Gao et al. (1993), ou seja, para a determinação da relação das intensidades das feiçôes dos minerais, adotou-se a média dos valores de reflectância dos pontos de início e fim da feição, menos a média dos pontos de absorção ou de mínima reflectância (Baptista 2006).

Já o índice $\mathrm{RCGb}_{\text {scale }}$ nada mais é que a relação caulinitagibbsita, ou seja, um índice proporcional ao Ki, desenvolvido a partir da modelagem sobre as imagens scale obtidas a partir da aplicação da classificação SFF. Isso torna possível a separação espacial dos minerais por meio do ajuste de mínimos quadrados entre os espectros de cada pixel e o espectro de referência de uma biblioteca espectral.

\section{Teste estatístico não paramétrico de Mann-Whitney (teste U)}

As estatísticas não paramétricas são técnicas de inferência que podem ser utilizadas como distribuiçôes de resultados que não obedecem aos parâmetros da curva normal. O método não paramétrico apenas mede a variabilidade dos resultados de forma indireta (Conover 1999).

O teste U é uma alternativa para o teste $t$ de Student com o objetivo de comparar as médias de duas amostras independentes. Os únicos pressupostos exigidos para a aplicação do teste U são a independência, a aleatoriedade e que as variáveis em análise sejam numéricas ou ordinais (Conover 1999).

As seguintes etapas foram seguidas para a realização do estudo:
1. Aquisição, no site WIST ECHO, dos dados de radiância L1A e L1B e dos produtos sob demanda AST_07 e AST_07XT de São João d'Aliança (GO), de 24/10/2001;

2. Por recomendação do manual do Envi (2009), realizou-se a reamostragem espacial de 30 para $15 \mathrm{~m}$ e a combinação das bandas do SWIR com as bandas do VNIR, para a aplicação do FLAASH e do QUAC. Com a intenção de padronização, a reamostragem espacial foi aplicada em todos os processamentos;

3. Aplicação dos módulos CRSTK30, FLAASH e QUAC. Nos processamentos com as duas correçôes, o CRSTK30 foi aplicado primeiramente, pois o tipo de dado utilizado na correção atmosférica difere do utilizado na correção crosstalk. Na primeira correção utiliza-se a imagem L1A (dados brutos), enquanto que na segunda o dado utilizado é o L1B radiância no nível do sensor;

4. Com base no fato de Baptista (2001) ter desenvolvido o $\mathrm{RCGb}_{\text {scale }}$ a partir dos espectros da biblioteca JPL, escolheram-se os espectros dos minerais Kaolinite Well-Ordered PS-1A e Gibbsite Synthetic OH-3A dessa mesma biblioteca espectral, os quais foram reamostrados para o ASTER;

5. Aplicação do módulo (SFF) para obtenção das imagens scale dos minerais;

6. Aplicação do índice espectral RCGb ${ }_{\text {scale }}$, por meio da expressão $\mathrm{b}_{1} /\left(\mathrm{b}_{1}+\mathrm{b}_{2}\right)$, inserida no módulo band math, em que $\mathrm{b}_{1}$ referiu-se à imagem scale kaolinite $\mathrm{e} \mathrm{b}_{2}$, à imagem scale gibbsite;

7. Fatiamento dos valores do RCGb $b_{\text {scale }}$ nos intervalos $(0,25-0,50) ;(0,50-0,75)$ e $(0,75-1,00)$;

8. Traçado do transecto de acordo com os pontos coletados em campo (Baptista 2001);

9. Seleção dos 13 pontos do transecto;

10. Aplicação do teste U;

11. Análise dos resultados segundo as quatro abordagens pré-estabelecidas, sendo essas: comparação do comportamento dos transectos do $\mathrm{RCGb}_{\text {scale }}$ e do $\mathrm{RCGb}_{\mathrm{ATG}}$, análise

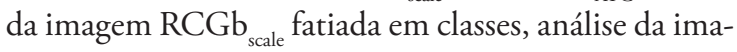
gem $\mathrm{RCGb}_{\text {scale }}$ e análise da similaridade estatística entre os valores dos transectos do RCGb scale $_{\text {e do }} \mathrm{RCGb}_{\mathrm{ATG}}$ ' por meio do teste estatístico de Mann Whitney.

Foram testados os seguintes processamentos:

1. imagem de radiância L1A (dados brutos) sem correção crosstalk e sem correção atmosférica;

2. imagem de radiância L1B (radiância no nível do sensor) sem correção crosstalk e sem correção atmosférica;

3. imagem de radiância L1B convertida para reflectância do topo da atmosfera (Flat Field), sem correçáo crosstalk e sem correção atmosférica; 
4. imagem de radiância L1B convertida para reflectância do topo da atmosfera (Flat Field), com correção crosstalk e sem correção atmosférica;

5. imagem de radiância L1A convertida para reflectância de superfície (FLAASH) sem correção crosstalk e com correção atmosférica;

6. imagem de radiância L1A convertida para reflectância de superfície (QUAC) sem correção crosstalk e com correção atmosférica;

7. imagem de radiância $\mathrm{L} 1 \mathrm{~B}$ convertida para reflectância de superfície (FLAASH) com correção crosstalk (CRSTK30) e com correção atmosférica;

8. imagem de radiância $\mathrm{L} 1 \mathrm{~B}$ convertida para reflectância de superfície (QUAC) com correção crosstalk (CRSTK30) e com correção atmosférica;

9. imagem de reflectância de superfície (AST_07) sem correção crosstalk e com correção atmosférica;

10. imagem de reflectância de superfície (AST_07XT) com correção crosstalk e com correção atmosférica.

\section{RESULTADOS E DISCUSSÃO}

Considerando que os dados da imagem de radiância foram normalizados por meio da remoção do contínuo espectral e que as feiçôes espectrais dos minerais, que são eventos quânticos, estão nos dados de radiância e não nos de irradiância, sua comparação com feiçóes de espectros que foram normalizados pelo mesmo método é possível. Tais dados variam em percentual e não mais em $\mathrm{W} / \mathrm{m}^{2}$.sr.um. Se não houvesse normalização, de fato as unidades seriam diferentes; porém, como os dados foram normalizados quando da classificação SFF em imagens de radiância, não representa impedimento à análise.

Após a realização dos processamentos, estabeleceu-se a seguinte classificação discricionária da importância das análises, com o objetivo de identificar o processamento mais adequado para o estudo:

- Análise do comportamento dos transectos, considerada a mais relevante por fornecer os valores dos pontos coletados no transecto e a sua tendência;

- Análise da imagem $\mathrm{RCGb}_{\text {scale }}$ fatiada em classes variando de 0 a 0,25 , de 0,25 a 0,5 , de 0,5 a 0,75 , e de 0,75 a 1,0 , por apresentar a classificaçáo dos valores do transecto em intervalos, possibilitando assim uma melhor identificação da relação mineralógica;

- Análise da imagem em tons de cinza, por apresentar a predominância dos ambientes;

- Análise estatística, por ter o papel complementar. Considerou-se, para a aplicaçáo do teste $\mathrm{U}$, o valor do $\mathrm{U}_{\text {tab }}=45$, de acordo com a tabela de valores críticos $\alpha=0,05$, para o tamanho de amostra $=13$, e como condicionantes: se $\mathrm{U}_{\text {calc }} \geq \mathrm{U}_{\text {tab }}$, entáo RCGb scale $_{\text {e }}$ e $\mathrm{RCGb}_{\text {ATG }}$ não apresentaram similaridade estatística; se $\mathrm{U}_{\text {calc }}<\mathrm{U}_{\mathrm{tab}}$, então RCGb scale $_{\text {e }}$ RCGb $\mathrm{ATG}_{\text {TG }}$ apresentaram similaridade estatística.

Isso levou ao ranqueamento dos resultados dos processamentos apresentado na Tab. 4.

Com relação aos transectos, os processamentos 1 e 7 foram considerados inadequados, pois ambos se apresentaram como retas uniformes sem apresentar a variabilidade da relação caulinita/gibbsita existente na área. Esse comportamento uniforme pode estar relacionado à natureza dos dados L1A, por manterem suas características originais, assim como não terem sido modificados pela aplicação dos coeficientes radiométricos, geométricos e os coeficientes para correção do erro de paralaxe do SWIR. No caso do processamento 7 , isso pode estar relacionado à combinação do algoritmo FLAASH com o módulo CRSTK30, não permitindo a identificação da relação mineralógica.

$\mathrm{Na}$ análise da imagem $\mathrm{RCG}_{\text {scale }}$ fatiada, foram considerados inadequados os processamentos 5, 6 e 7 . O processamento 5 apresentou uma faixa ruidosa na porção direita da imagem, além de a classe de 0,75 a 1,0 apresentar distribuição espacial reduzida, e a de 0,5 a 0,75 expandida. É importante ressaltar que nos processamentos considerados adequados, a classe de 0,75 a 1,0 mostrou-se predominante. No processamento 6, a imagem fatiada apresentou maior mistura das classes, dificultando a identificação da relaçáo mineralógica. No processamento 7, apresentou mistura das classes, dificultando a identificação da relação mineralógica.

$\mathrm{Na}$ análise da imagem $\mathrm{RCG}_{\text {scale }}$ em tons de cinza, os processamentos considerados inadequados formam os mesmos da imagem fatiada, o que era de se esperar, pois uma é o reagrupamento da outra. No processamento 5, o RCGb apresentou homogeneidade dos tons de cinza, além da faixa escura atípica salientada anteriormente, atribuída a possíveis erros durante a aplicação do FLAASH, o que dificultou a identificação dos ambientes cauliníticos e gibbsíticos. No 6, apresentou pouca variação dos tons de cinza e no 7 , apresentou mistura dos tons de cinza.

Finalmente, o teste U de Mann-Whitney foi o que apresentou o maior número de processamentos considerados como inadequados, os processamentos 1, 2, 3, 4, 6 e 10, pois não indicou similaridade estatística entre os valores dos transectos do $\mathrm{RCGb}_{\text {scale }}$ e do $\mathrm{RCGb}_{\mathrm{ATG}}$, ou os obtidos com as amostras de solo coletadas no campo.

É importante esclarecer que no teste estatístico de MannWhitney os valores de $U$ calculados avaliam o grau de entrelaçamento dos dados dos dois grupos após a ordenação. A maior separação dos dados, em conjunto, indica que as amostras são distintas, rejeitando-se a hipótese de igualdade 
Tabela 4. Ranqueamento dos resultados dos processamentos

\begin{tabular}{|c|c|c|c|c|}
\hline \multirow{2}{*}{ Processamento } & \multirow{2}{*}{ Transecto } & \multicolumn{2}{|c|}{ Imagem } & \multirow{2}{*}{ Teste U } \\
\hline & & Fatiamento & Cinza & \\
\hline $\begin{array}{l}\text { 9: Produto sob demanda ASTER AST-07 - } \\
\text { imagem de reflectância sem correção crosstalk } \\
\text { e com correção atmosférica }\end{array}$ & Adequado & Adequado & Adequado & Adequado \\
\hline $\begin{array}{l}\text { 8: Imagem L1B convertida para reflectância, } \\
\text { com correção crosstalk, por meio do programa } \\
\text { CRSTK30 e com correção atmosférica, por meio } \\
\text { da aplicação do algoritmo QUAC }\end{array}$ & Adequado & Adequado & Adequado & Adequado \\
\hline $\begin{array}{l}\text { 10: Produto sob demanda ASTER AST-07XT - } \\
\text { imagem de reflectância com correção crosstalk } \\
\text { e com correção atmosférica }\end{array}$ & Adequado & Adequado & Adequado & Inadequado \\
\hline $\begin{array}{l}\text { 3: Imagem L1B convertida para reflectância, } \\
\text { por meio do módulo Flat Field, sem correção } \\
\text { crosstalk e sem correção atmosférica }\end{array}$ & Adequado & Adequado & Adequado & Inadequado \\
\hline $\begin{array}{l}\text { 4: Imagem L1B convertida para reflectância, } \\
\text { por meio do módulo Flat Field, com correção } \\
\text { crosstalk, por meio do programa CRSTK30 e } \\
\text { sem correção atmosférica }\end{array}$ & Adequado & Adequado & Adequado & Inadequado \\
\hline $\begin{array}{l}\text { 2: Imagem de radiância L1B (radiância } \\
\text { no nível do sensor) sem correção crosstalk } \\
\text { e sem correção atmosférica }\end{array}$ & Adequado & Adequado & Adequado & Inadequado \\
\hline $\begin{array}{l}\text { 5: Imagem de radiância L1A convertido para } \\
\text { reflectância, sem correção crosstalk e com } \\
\text { correção atmosférica, por meio da aplicação } \\
\text { do algoritmo FLAASH }\end{array}$ & Adequado & Inadequado & Inadequado & Adequado \\
\hline $\begin{array}{l}\text { 6: Imagem de radiância L1A convertida } \\
\text { para reflectância, sem correção crosstalk } \\
\text { e com correção atmosférica por meio da } \\
\text { aplicação do algoritmo QUAC }\end{array}$ & Adequado & Inadequado & Inadequado & Inadequado \\
\hline $\begin{array}{l}\text { 1: Imagem de radiância L1A (dados brutos) sem } \\
\text { correção crosstalk e sem correção atmosférica }\end{array}$ & Inadequado & Adequado & Adequado & Inadequado \\
\hline $\begin{array}{l}\text { 7: Imagem L1B convertida para reflectância, } \\
\text { com correção crosstalk, por meio do programa } \\
\text { CRSTK30 e com correção atmosférica por meio } \\
\text { da aplicação do algoritmo FLAASH }\end{array}$ & Inadequado & Inadequado & Inadequado & Adequado \\
\hline
\end{tabular}

das medianas, é o caso do resultado desses processamentos considerados inadequados.

No entanto, é importante lembrar que a finalidade do sensoriamento remoto está em fornecer dados digitais, o quão próximo da realidade for possível, ou seja, o quão próximo pode-se chegar do produto produzido pelos sensores naturais (os olhos).

Portanto, neste estudo, a análise visual tem predomínio sobre os resultados da análise estatística, a qual tem papel de complementação, não perdendo, porém, a sua importância, pois a natureza não se prende aos números.

Após o ranqueamento dos resultados dos processamentos, visando compreendê-los visualmente, foram apresentados apenas o melhor e o pior processamento. Como se pôde observar na Tab. 4, no ranqueamento, o processamento 7 apresentou-se inadequado nas três primeiras análises e os processamentos 9 e 8 apresentaram-se adequados nas quatro análises. Escolheu-se o processamento 7 como o pior e o 9, como o melhor.

\section{Processamento 7: imagem de reflectância convertida a partir dos dados 11 b com correçáo crosstalk, por meio do programa CRSTK30 e com correçáo atmosférica por meio da aplicação do algoritmo FLAASH}

O transecto do RCGb scale-FLAASH $_{\text {apresentou valores }}$ muito próximos entre si e superestimados em relação aos valores do $\mathrm{RCGb}_{\mathrm{ATG}}$, o que não possibilitou variação suficiente para promover um desenho similar ao do $\mathrm{RCGb}_{\mathrm{ATG}}$, e sim um comportamento uniforme aplainado. Isso pode 
estar relacionado à combinação do algoritmo FLAASH com o módulo CRSTK30, não permitindo a identificação da relação mineralógica. A Fig. 3 apresenta os transectos do $\mathrm{RCGb}_{\text {scale-FLAASH }}$ e do RCGb ATG $_{\text {. }}$.

A imagem fatiada em classes do RCGb scale-FLAASH apresentou mistura das classes, dificultando a identificação da relação mineralógica. A Fig. 4 apresenta a imagem do RCGb

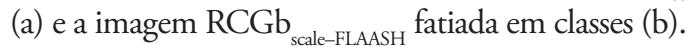

A imagem RCGb $b_{\text {scale-FLAASH }}$ não proporcionou resultado positivo na identificação dos ambientes cauliníticos e gibbsíticos, pois apresentou mistura dos tons de cinza.

O teste estatístico forneceu resultado de similaridade

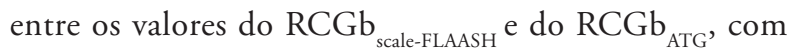
$\mathrm{U}_{\text {calc }}=0$. A Tab. 5 apresenta o resultado do teste $\mathrm{U}$.

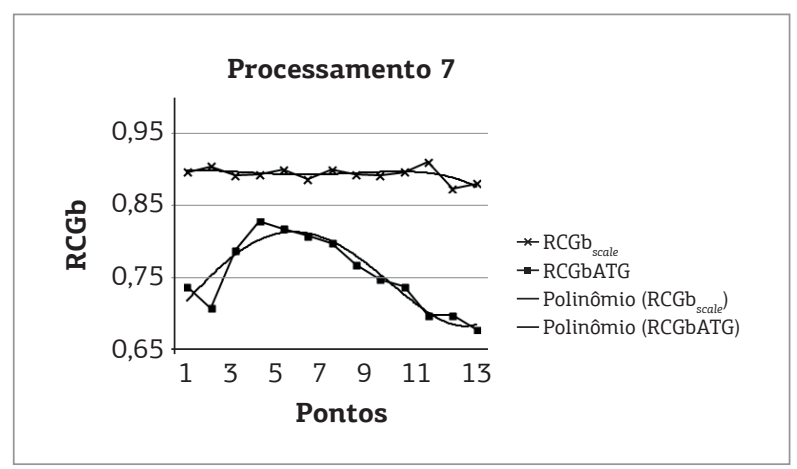

Figura 3. Comportamento dos transectos do $\mathrm{RCGb}_{\text {sca }}$ da imagem L1B de reflectância, com correção crosstalk (CRSTK30) e com correção atmosférica (FLAASH) e do RCGb ${ }_{\text {ATG }}$.

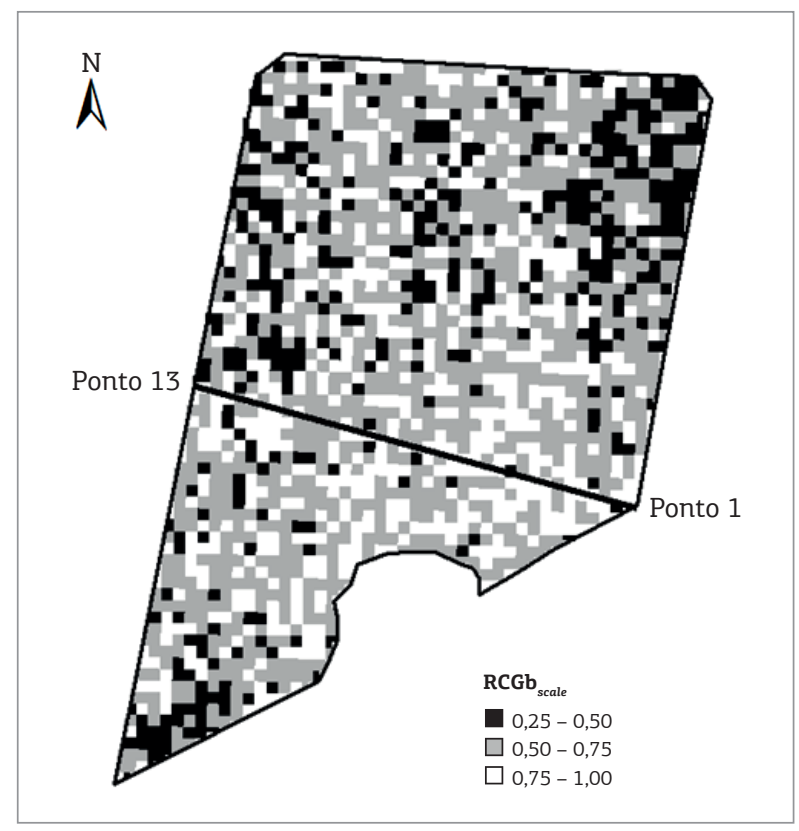

Figura 4. Imagem $\mathrm{RCGb}_{\text {scale-FLAASH }}$ fatiada em classes.
Processamento 9: produto sob demanda ASTER AST_07 - imagem de reflectância sem correçáo crosstalk e com correção atmosférica

$\mathrm{O}$ transecto do $\mathrm{RCGb}_{\text {scale }}$ apresentou comportamento similar ao do $\mathrm{RCGb}_{\mathrm{ATG}}$, apesar da suavização dos valores entre os pontos 3 e 11 e da uniformidade verificada entre os pontos 3 e 8. A Fig. 5 apresenta os transectos do RCGb scale $_{\text {e }}$ e do $\mathrm{RCGb}_{\mathrm{ATG}}$.

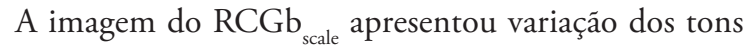
de cinza que possibilitaram a identificação dos ambientes cauliníticos e gibbsíticos.

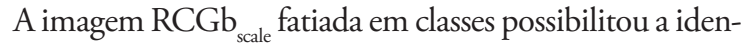
tificação da relação mineralógica, por meio da distribuição das classes, tendo início com valores da classe 0,25 - 0,50 seguido da classe $0,50-0,75$, da classe $0,75-1,00$, retornando à classe 0,50-0,75 e finalizando com a classe 0,25-0,50. A Fig. 6 apresenta a imagem do $\mathrm{RCGb}_{\text {scale }}$ (a) e a imagem $\mathrm{RCGb}_{\text {scale }}$ fatiada em classes (b).

$O$ resultado do teste $U$ apresentou similaridade estatística entre os valores do $\mathrm{RCGb}_{\text {scale }}$ e do $\mathrm{RCGb}_{\mathrm{ATG}}$, com $\mathrm{U}_{\text {calc }}=44,5$. A Tab. 6 apresenta o resultado do teste $U$.

Tabela 5. Aplicação do teste de Mann-Whitney sobre os valores do RCGb

\begin{tabular}{l|c|c}
\hline \multicolumn{3}{c}{ Análise não paramétrica de Mann-Whitney } \\
\hline Resultado & RCGb $_{\text {scale }}$ & RCGb $_{\text {ATG }}$ \\
\hline Tamanho da amostra & 13 & 13 \\
\hline Soma dos Postos (Ri) & 260,0 & 91,0 \\
\hline Mediana & 0,90 & 0,75 \\
\hline$U_{\text {calc }}$ & \multicolumn{2}{|c}{0,00} \\
\hline valor p (unilateral) & \multicolumn{2}{|c}{$<0,0001$} \\
\hline valor p (bilateral) & \multicolumn{2}{c}{$<0,0001$} \\
\hline
\end{tabular}

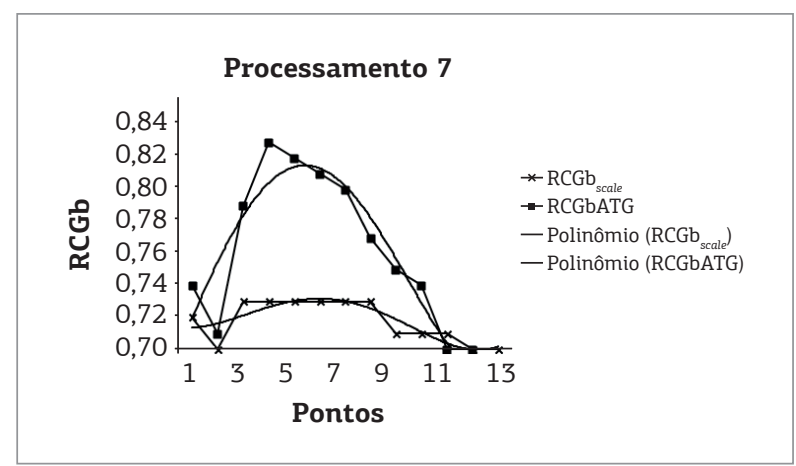

Figura 5. Comportamento dos transectos do $\mathrm{RCGb}_{\text {scale }}$ em produto sob demanda AST_07 e do RCGb ${ }_{\mathrm{ATG}}$. 


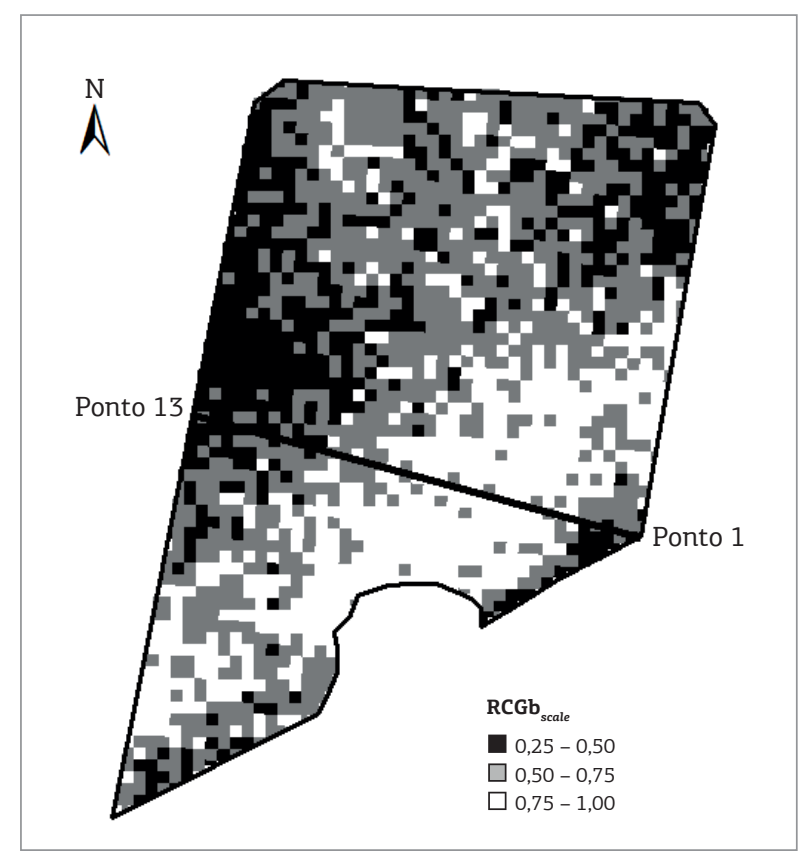

Figura 6. Imagem RCGb ${ }_{\text {scale }}$ fatiada em classes.

Concluiu-se que o produto sob demanda AST_07 sem correção crosstalk e com correção atmosférica é adequado na identificação da relação mineralógica em solos da área estudada, pois foi considerado como adequado nas quatro análises.

\section{CONCLUSÕES}

Os efeitos crosstalk e atmosféricos influenciaram no comportamento uniforme do transecto do $\mathrm{RCGb}_{\text {scale }}$ apenas nos dados de radiância L1A (dados brutos). A correção crosstalk e a correção atmosférica, por meio do FLAASH, quando combinadas, também influenciaram no comportamento uniforme do transecto do RCGb $\mathrm{scale}_{\text {. }}$ Os efeitos crosstalk e atmosféricos não impediram a identificação dos ambientes em imagens de radiância e de reflectância $\mathrm{RCGb}_{\text {scale }}$, assim
Tabela 6. Aplicação do teste de Mann-Whitney sobre os valores do $R C G b_{\text {scale }}$ e do $R C G b_{\text {ATG }}$

\begin{tabular}{l|c|c}
\hline \multicolumn{3}{c}{ Análise não paramétrica de Mann-Whitney } \\
\hline Resultado & RCGb $_{\text {scale }}$ & RCGb $_{\text {ATG }}$ \\
\hline Tamanho da amostra & 13 & 13 \\
\hline Soma dos Postos (Ri) & 135,5 & 215,5 \\
\hline Mediana & 0,72 & 0,75 \\
\hline$U_{\text {calc }}$ & \multicolumn{2}{|c}{44,50} \\
\hline valor p (unilateral) & \multicolumn{2}{|c}{0,0201} \\
\hline valor p (bilateral) & \multicolumn{3}{|c}{0,0402} \\
\hline
\end{tabular}

como a identificação da relação mineralógica. A identificação dos ambientes e da relação mineralógica foi prejudicada na imagem $\mathrm{RCGb}_{\text {scale }}$ e na imagem $\mathrm{RCGb}_{\text {scale }}$ fatiada em classes com a correçáo atmosférica aplicada por meio do FLAASH e do QUAC. A identificação dos ambientes e da relação mineralógica foi prejudicada na imagem $\mathrm{RCGb}_{\text {scale }}$ quando se combinou a correção crosstalk com a correção atmosférica por meio do FLAASH. A aplicação da correção atmosférica forneceu melhores resultados de similaridade estatística entre os valores $\mathrm{RCGb}_{\text {scale }}$ e do $\mathrm{RCGb}_{\mathrm{ATG}}$ quando comparados aos demais resultados. A correção crosstalk forneceu melhores resultados de similaridade estatística apenas quando combinada à correção atmosférica por meio do FLAASH e do QUAC.

De acordo com a classificação das análises, concluiu-se que, para a investigação da relação caulinita/(caulinita+gibbsita) na área de estudo em São João d'Aliança (GO), o produto sob demanda AST_07 e a imagem de reflectância L1B, com correção crosstalk (CRSTK30) e com correção atmosférica (QUAC) são as mais adequadas, nessa ordem.

Novos trabalhos devem ser incentivados visando testar os efeitos dos pré-processamentos de ajustes radiométricos na análise das relaçóes mineralógicas por meio de dados ASTER.

\section{REFERÊNCIAS}

Abrams M., Hook S. \& Ramachandran B. 2007. Aster user handbook: version 2. California: jpl - jet propulsion laboratory. Disponível em: http://asterweb.jpl.nasa.gov/content/03_data/04_ Documents/aster_user_guide_v2.pdf. Acessado em 20/05/2012.

Abrams M. \& Kahle A., 2000. The Advanced Spaceborne Thermal Emission and Reflection Radiometer (ASTER). 2nd EARSeL Workshop on Imaging Spectroscopy, held in Enschedde, Netherlands.

Advanced Spaceborne Thermal Emission and Reflection Radiometer (ASTER). SWIR. Disponível em: http://asterweb.jpl.nasa.gov/swir.asp. Acessado em 20/05/2012.
Conover W.J. 1999. Practical nonparametric statistics. 3rd ed. New York: John Wiley \& Sons, Inc.

Baptista, G.M.M., 2012. Processamento de Dados Hiperespectrais. In: Meneses \& Almeida (org). Introdução ao Processamento de Imagens de Sensoriamento Remoto. Brasília: UnB/CNPq, p. 221-238.

Baptista G.M.M., Correa R.S., Santos P.F., Madeira Netto J.S. \& Meneses P.R. 2011. Use of Imaging Spectroscopy for Mapping and Quantifying the Weathering Degree of Tropical Soils in Central Brazil. Applied and Environmental Soil Science. Special Issue Remote Sensing of Soil, p. 1-7. 
Baptista G.M.M., Correa R.S., Torres M.G., Bias E.S., Resende M.G., Ribeiro R.J.C. \& Silva D.J. 2007. Identificação de relação mineral Caulinita/Gibbsita em solos tropicais, por meio dos dados SWIR do sensor ASTER. In: INPE, XIII Simpósio Brasileiro de Sensoriamento Remoto, Anais, p. 7043-7050.

Baptista G.M.M. 2006. Sensoriamento Remoto Hiperespetral: o novo paradigma nos estudos de solos tropicais. Brasília: Editora Universa, 212 p.

Baptista G.M.M. \& Madeira Netto J.S. 2001. RCGb Index: a tool for mapping the weathering degree of the tropical soils in Brazil. In: NASA, The AVIRIS Earth Science and Applications Workshop, Proceedings, p. 1-10.

Baptista G.M.M. 2001. Mapeamento e quantificação da relação Caulinita/(Caulinita+Gibbsita) de solos tropicais, por meio dos dados do sensor AVIRIS (JPL/NASA). Tese (doutorado). Instituto de Geociências Aplicadas, Universidade de Brasília, 278 p.

Empresa Brasileira de Pesquisa Agropecuária (Embrapa). 1999. Sistema brasileiro de classificação de solos. Rio de Janeiro: Embrapa Solos, $412 \mathrm{p}$.

Environment for Visualizing Images (ENVI). 2009. User's Guide: Atmospheric Correction Module: QUAC and FLAASH. ESRI

EARTH REMOTE SENSING DATA ANALYSIS CENTER (ERSDAC). 2003. Crosstalk correction program user's guide. Tokyo: ERSDAC, 2003. 21 p. Disponivel em: http://www.gds.ASTER.ersdac.or.jp/gds www2002/service_e/u.tools_e/cross/GUIDEED.PDF. Acessado em 20/05/2012

Faria A. 1995. Estratigrafia e Sistemas Deposicionais do Grupo Paranoá nas Áreas de Cristalina, Distrito Federal e São João d'Aliança - Alto Paraíso de Goiás. Tese (doutorado), Instituto de Geociências, Universidade de Brasília, Brasília, 199 p.

Galvão L.S., Pizarro M.A. \& Epiphanio, J.C.N. 2001. Variations in reflectance of tropical soils: spectral-chemical compositions relationships from AVIRIS data. Remote Sensing Environment. 75:245-255.
Galvão L.S, Vitorello I. \& Formaggio A.R. 1997 Relationships of spectral reflectance and color among surface and sudsurface horizons of tropical soil profiles. Remote Sensing Environment. 61:24-33.

Gao B.C., Goetz Heidebrecht K.B. \& Goetz A.F.H. 1993. Derivation of scaled surface reflectance from AVIRIS data. Remote Sensing Environment. 44:165-178.

Grove C.I., Hook S.J. \& Taylor E.D. 1992. Laboratory reflectance of 160 minerals, 0,4 to 2,5 micrometers. Passadena: National Aeronautics and Space Administration, $355 \mathrm{p}$.

Jensen J.R. 2009. Sensoriamento remoto do ambiente: uma perspectiva em recursos terrestres. São José dos Campos, SP: Parêntese.

Kruse F.A. 2004. Comparison of ATREM, ACORN, and FLAASH atmospheric corrections using low-altitude AVIRIS data of Boulder, CO. Summaries of 13th JPL Airborne Geoscience Workshop, Jet Propulsion Lab, Pasadena, CA.

Mackenzie R.C. 1982. Thermoanalytical methods in clay studies. In: Fripiat J. (ed.). Advanced techniques for clay mineral analysis. Development in Sedimentology, 34:5-29.

Madeira Netto J.S. 2001. Comportamento espectral dos solos. In: Meneses, P.R. \& Madeira Netto, J.S. Sensoriamento remoto: reflectância dos alvos naturais. Brasília: UnB/EMBRAPA, p. 127-154.

Meneses P.R. 2003. Fundamentos de radiometria óptica espectral. In: Meneses, P.R. \& Madeira Netto, J.S. Sensoriamento remoto: reflectância de alvos naturais. Brasília: UnB/ EMBRAPA, p. 15-60.

Meneses P.R. \& Almeida T. (org.) 2012. Introdução ao Processamento de Imagens de Sensoriamento Remoto. Brasília: UnB/CNPq.

USGS. The Land Processes Distributed Active Archive Center. Disponível em: https://lpdaac.usgs.gov/. Acessado em 04/03/2013.

$\overline{\text { Arquivo digital disponível on-line no site www.sbgeo.org.br }}$ 